\title{
Rapid Automated Preparation for Serial Block Face Scanning Electron Microscopy
}

Steven L Goodman ${ }^{1 *}$, Emily K Benson ${ }^{2}$, Jay M Campbell ${ }^{1,3}$, and Grahame J Kidd ${ }^{3}$

${ }^{1}$ Microscopy Innovations LLC, Marshfield, WI, USA.

2. Renovo Neural Inc., Cleveland, OH, USA.

3. University of Wisconsin-Madison School of Medicine and Public Health, Madison, WI, USA.

*Corresponding author: Steven.Goodman@MicroscopyInnovations.com

By providing automated acquisition of large volume electron microscope image stacks, serial block-face electron microscopy (SBEM) creates exciting new possibilities for life science discovery. While many repetitive aspects of microscopy are now computer-controlled, sample preparation remains a hurdle. SBEM sample preparation uses chemical fixation, heavy metal staining, and resin embedding protocols based on those developed for TEM, plus additional toxic and reactive steps. While TEM preparation typically requires 1-2 days, nearly week-long SBEM protocols are used to provide the heavy metal staining and conductivity required for high-contrast back-scattered SEM imaging at 1-3 KeV.

We report herein an SBEM protocol for neurological tissue where all reagent exchanges were automatically performed in one working day with the mPrep ASP-1000 Automated Specimen Processor (ASP). The ASP enables high-speed preparation of biological tissues with rapid and repeated fluid exchanges that accelerate reagent diffusion into specimens, which can prepare tissues for TEM as rapidly as 1-3 hours [1]. For SBEM, a 13-hour hands-on manual protocol with 3-4 overnight treatments was accelerated into a 7.5-hour automated protocol, with only one overnight for resin curing.

Brain was excised from a perfusion-fixed 12-month old rat, then immersed and refrigerated in cacodylate-buffered glutaraldehyde-paraformaldehyde until subsequent preparation. Cortex and corpus collosum specimens (1-3 mm) were loaded into labeled mPrep/s capsules. Capsules were mounted onto the ASP and reagents were dispensed into microwell trays with light sensitive and reactive reagents tightly covered using microplate sealing foil (Fig. 1). The ASP executed the protocol (Fig. 2) by aspirating successive reagents into each capsule for the programmed time, breaching foil seals as needed. Agitation was provided by gentle flow reversals through the capsules every few seconds. Epoxy infiltrated specimens in capsules were removed from the ASP and cured overnight at $60 \mathrm{C}$. An FEI Teneo VolumeScope imaged blocks at $2.0 \mathrm{kV}, 0.1 \mathrm{nA}$ under high vacuum using the T1 detector. Images $\sim 60 \times 60$ um and 20 um deep ( 350 slices with $70 \mathrm{~nm}$ cuts) were acquired in $25 \mathrm{hr}$ runs. Figure 3 is a perspective projection SBEM image of a sample of cortex prepared with the ASP.

To compare preparation quality, samples from the same animal were prepared manually (Fig. 4a) and with the ASP (Fig. 4b). The evenness of myelin staining in both indicate good reagent penetration since lipid-rich myelin membranes are both a dense target of metal staining and a barrier to diffusion. Synaptic vesicle clarity (Figs. 4a-b) and discrimination of mitochondrial cristae against the dark mitochondrial matrix (Figs. 4a-b), were also comparable. Neither the ASP or the manually-prepared specimens exhibited common SBEM problems including brittle, cracking, or shrinking blocks, or blocks that produce shards during cutting. Overall, the ASP produced comparable preparations in much less time. This reduction in overall time and manual effort are clear advantages for ASP preparation. Additionally, the reproducibility of automation and the capability of programmable control to optimize protocols for different tissues and research needs are also benefits. Further, the ASP could also perform 
SBEM immunostaining, as demonstrated for TEM [2]. Finally, since the enclosed ASP either vents into or can be contained in a fume hood, experimenter exposure to noxious reagents may be reduced.

\section{References:}

[1] TE Strader et al., Microsc. Microanal 24 (2018), p. 1284.

[2] P Marques et al., Microsc. Microanal 24 (2018), p. 1300.

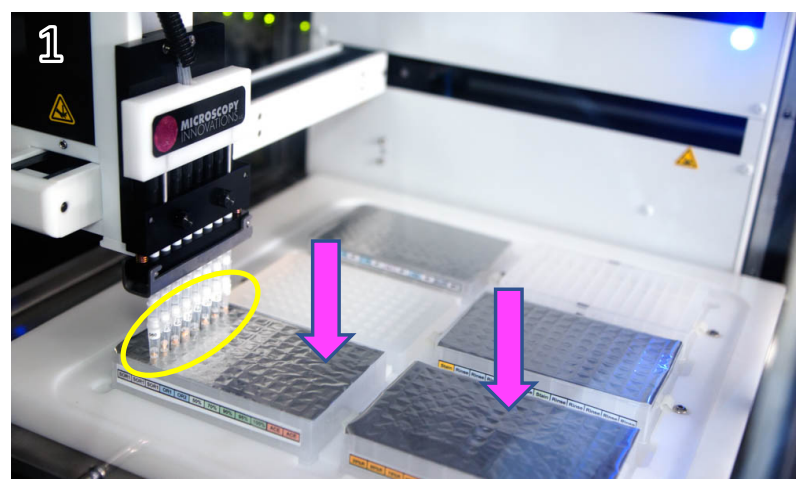

2 \begin{tabular}{|c|c|c|c|}
\hline Reagent & Exchanges & Agitations & Time (min) \\
\hline Glut/PFA & perfusion & & $5^{\circ}$ C until process \\
\hline Buffer Rinse & 3 & 90 & 3 \\
\hline Tannic acid & 1 & 450 & 15 \\
\hline $\mathrm{K}_{4}\left[\mathrm{Fe}(\mathrm{CN})_{6}\right]$ & 1 & 1800 & 60 \\
\hline $\mathrm{H} 2 \mathrm{O}$ & $5 \times 3$ & 450 & 15 \\
\hline $\mathrm{TCH}$ & 1 & 1800 & 60 \\
\hline $\mathrm{H} 2 \mathrm{O}$ & 1 & 90 & 3 \\
\hline $\mathrm{OsO} 4$ & 1 & 900 & 35 \\
\hline $\mathrm{H} 20$ & $5 \times 5$ & 450 & 25 \\
\hline $\mathrm{UrAc}$ & 1 & 1800 & 60 \\
\hline $\mathrm{H} 2 \mathrm{O}$ & $3 \times 2$ & 270 & 6 \\
\hline Lead Aspartate & 1 & 900 & 30 \\
\hline $\mathrm{H} 2 \mathrm{O}$ & $3 \times 5$ & 135 & 15 \\
\hline $25 \%$ EtOH & 1 & 110 & 10.5 \\
\hline $50 \%$ EtOH & 1 & 110 & 10.5 \\
\hline $75 \%$ EtOH & 1 & 110 & 10.5 \\
\hline $95 \%$ EtOH & 1 & 150 & 10.5 \\
\hline $100 \%$ EtOH & 1 & 150 & 10.5 \\
\hline Acetone & $2 \times 10$ & 540 & 10.5 \\
\hline $25 \%$ Resin & 1 & 300 & 10.5 \\
\hline $50 \%$ Resin & 1 & 300 & 10.5 \\
\hline $100 \%$ Resin & $3 \times 10$ & 360 & 31.5 \\
\hline Resin cure & 1 & & Overnight \\
\hline Total Automatic Processing Time & $\mathbf{7 h r} \mathbf{2 2}$ min \\
\hline
\end{tabular}
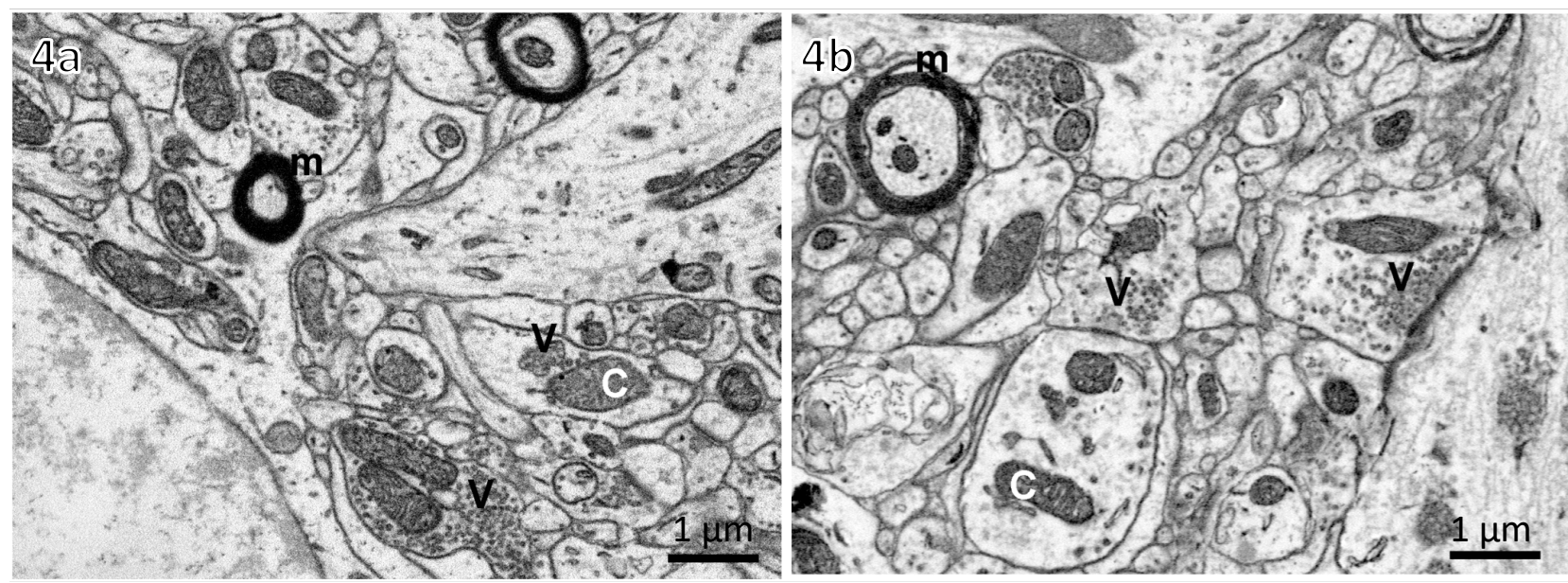

Figure 1. ASP-1000. Specimens in mPrep/s capsules (circled). Reagents in microwell plates (arrows).

Figure 2. Automated ASP protocol.

Figure 3. Cortex prepared with ASP. Perspective projection view from 35070 um thick slices.

Figure 4. Manually prepared cortex (4a) and ASP prepared cortex (4b) from same brain: Myelin (m), synaptic vesicles (v) mitochondrial cristae (c). 